\title{
The structure of an earthward propagating magnetic flux rope early in its evolution: comparison of methods
}

\author{
C. Möstl ${ }^{1,2}$, C.J. Farrugia ${ }^{3}$, H. K. Biernat ${ }^{1,2}$, S. A. Kiehas ${ }^{2}$, R. Nakamura ${ }^{2}$, V. V. Ivanova ${ }^{2}$, and Y. Khotyaintsev \\ ${ }^{1}$ Space Research Institute, Austrian Academy of Sciences, 8042 Graz, Austria \\ ${ }^{2}$ Institute for Physics, University of Graz, 8010 Graz, Austria \\ ${ }^{3}$ Space Science Center and Dept. of Physics, University of New Hampshire, Durham, NH 03824, USA \\ ${ }^{4}$ Swedish Institute of Space Physics, 75121 Uppsala, Sweden
}

Received: 20 October 2008 - Revised: 15 April 2009 - Accepted: 21 April 2009 - Published: 15 May 2009

\begin{abstract}
We analyze a magnetic signature associated with the leading edge of a bursty bulk flow observed by Cluster at $-19 R_{E}$ downtail on 22 August 2001. A distinct rotation of the magnetic field was seen by all four spacecraft. This event was previously examined by Slavin et al. (2003b) using both linear force-free modeling as well as a curlometer technique. Extending this work, we apply here single- and multi-spacecraft Grad-Shafranov (GS) reconstruction techniques to the Cluster observations and find good evidence that the structure encountered is indeed a magnetic flux rope and contains helical magnetic field lines. We find that the flux rope has a diameter of approximately $1 R_{E}$, an axial field of $26.4 \mathrm{nT}$, a velocity of $\approx 650 \mathrm{~km} / \mathrm{s}$, a total axial current of $0.16 \mathrm{MA}$ and magnetic fluxes of order $10^{5} \mathrm{~Wb}$. The field line twist is estimated as half a turn per $R_{E}$. The invariant axis is inclined at $40^{\circ}$ to the ecliptic plane and $10^{\circ}$ to the GSM equatorial plane. The flux rope has a force-free core and nonforce-free boundaries. When we compare and contrast our results with those obtained from minimum variance, singlespacecraft force-free fitting and curlometer techniques, we find in general fair agreement, but also clear differences such as a higher inclination of the axis to the ecliptic. We further conclude that single-spacecraft methods have limitations which should be kept in mind when applied to THEMIS observations, and that non-force-free GS and curlometer techniques are to be preferred in their analysis. Some properties we derived for this earthward- moving structure are similar to those inferred by Lui et al. (2007), using a different approach, for a tailward-moving flux rope observed during the expansion phase of the same substorm.
\end{abstract}

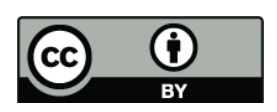

Correspondence to: C. Möstl (christian.moestl@oeaw.ac.at)
Keywords. Magnetospheric physics (Magnetotail; Storms and substorms) - Space plasma physics (Magnetic reconnection)

\section{Introduction}

Magnetic flux ropes occur in various contexts in space plasmas. In the Earth's magnetotail during the substorm expansion phase they have been observed propagating both earthward ("bursty bulk flow (BBF) type") and tailward ("plasmoid type"), as classified by Slavin et al. (2003a). In recent years, many studies focused on the "BBF-type" (e.g. Slavin et al., 2003b; Zong et al., 2004; Henderson et al., 2006; Hasegawa et al., 2007; Lui et al., 2007; Walsh et al., 2007; Zhang et al., 2007), especially since multi-point in situ measurements became available in the near tail $\left(X_{\mathrm{GSE}}>-25 R_{E}\right)$ from the Cluster mission. Their importance lies as much in their ability to transport magnetic flux, currents and plasma from the magnetotail towards Earth, as in the fact that their internal structure may offer valuable clues on their formation mechanism. It is the purpose of this paper to investigate the structure of a specific BBF-type flux tube using CLUSTER observations and Grad-Shafranov reconstruction methods and to draw conclusions on the limitations of singlespacecraft techniques. This ia an important issue in the age of the THEMIS mission (Angelopoulos, 2008).

A model which is widely used considers a given flux tube as a linear force-free configuration (i.e. one satisfying $\boldsymbol{j}=\alpha \boldsymbol{B})$ of constant alpha and of local straight cylindrical geometry, a solution of which was given in terms of Bessel functions by Lundquist (1950). In this model the flux rope field winds around the axis with a field pitch which decreases

Published by Copernicus Publications on behalf of the European Geosciences Union. 


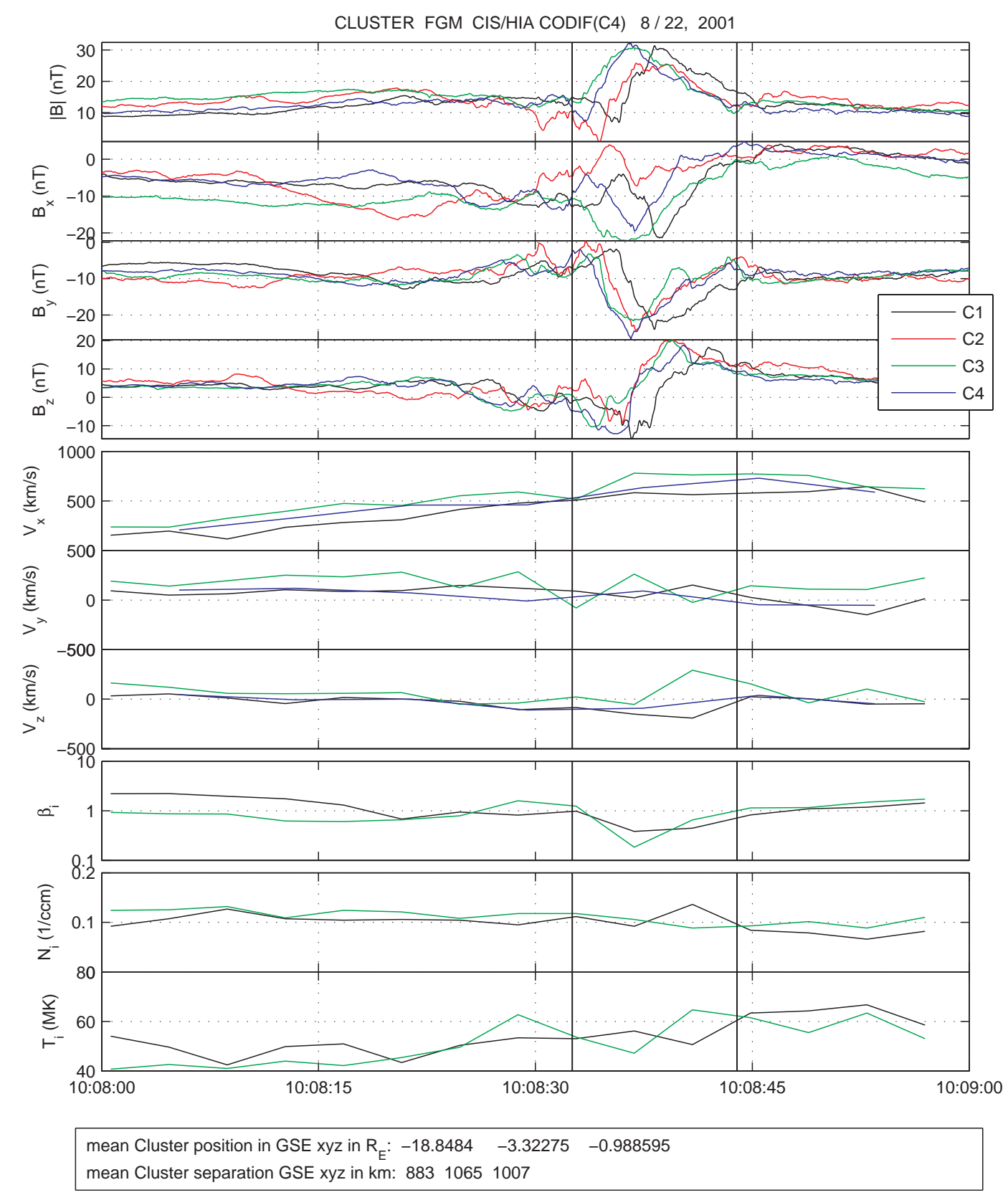

Fig. 1. Cluster magnetic field and ion/proton data for 22 August 2001, 10:08:00-10:09:00 UT. From top to bottom: Magnetic field magnitude, magnetic field components (GSE), proton bulk velocity (GSE), ion beta, ion density and ion temperature. Plasma parameters are from CIS/HIA for $\mathrm{C} 1$ and $\mathrm{C} 3$, the proton velocity from HIA for $\mathrm{C} 1 / \mathrm{C} 3$ and from CODIF for $\mathrm{C} 4$. The vertical lines delimit the interval 10:08:3310:08:44 UT used for the reconstruction.

steadily to zero on the boundary (where the field lines are circular; e.g. Burlaga, 1988; Lepping et al., 1990). In the magnetotail context, this solution is then fitted to in-situ measurements of the magnetic field profile, consisting of a bipolar $B_{z}$ signature accompanied by a monopolar deflection in $B_{y}$. Slavin et al. (2003a) found that about $60 \%$ of both BBF and plasmoid-type flux ropes agreed reasonably well with the
Lundquist linear force-free model. Further, the authors suggested that magnetic flux ropes arise from multiple X-line reconnection (MRX; e.g. Schindler, 1974; Lee, 1995). In that case the internal field is expected to have a rather smooth helical structure, even though it can depart strongly from the force-free state especially at the boundaries (Slavin et al., 2003b; Henderson et al., 2006), due to interaction with the 
surrounding plasma. Note that the other $40 \%$ could not be so modeled. Significantly for what follows, Lui et al. (2007) argued that a specific plasmoid-type flux rope observed by Cluster somewhat earlier than the event we study had a complex internal field line structure and was also inconsistent with force-free models.

A different approach for modeling flux ropes consists in numerically integrating the Grad-Shafranov (GS) equation to produce maps of the magnetic field and plasma pressure. The GS equation is valid for describing time-independent and $2 \frac{1}{2}$-dimensional (i.e. with an invariance direction) magnetic structures in space plasmas. Originally introduced for studies of the magnetopause during traversals of this boundary (Hau and Sonnerup, 1999), it has been applied to flux ropes such as magnetic clouds (e.g. Hu and Sonnerup, 2002; Hu et al., 2005; Möstl et al., 2008, 2009), dayside flux transfer events (Sonnerup et al., 2004; Hasegawa et al., 2006), and magnetic flux ropes and field aligned currents associated with BBFs in the magnetotail (Hasegawa et al., 2007; Zhang et al., 2007; Snekvik et al., 2007). The technique does not assume forcefree conditions nor does it prescribe a geometry (e.g. circular field lines), and thus relaxes two important constraints of the classical fitting technique, while invariance along a particular direction (the flux rope axis) is still assumed and tested a posteriori. Additionally, a multi-spacecraft GS technique has been developed (Sonnerup et al., 2004; Hasegawa et al., 2005). It has several advantages over the single-spacecraft GS technique: (1) information from multiple sites is used to assess the validity of the integrated magnetic field maps, (2) a combined magnetic field map is created which best fits all available observations, and (3) inertial effects are incorporated in a low order approximation (Hasegawa et al., 2005). What is not implemented at present but may be of importance in the magnetotail are effects arising from expansion, HallMHD, temperature anisotropy, and strong field aligned flows; see Sonnerup et al. (2006) and Sonnerup and Teh (2008) for recent developments.

In this paper we apply both, single (in the version of $\mathrm{Hu}$ and Sonnerup, 2002) and multi-spacecraft GS reconstruction methods (as in Sonnerup et al., 2004), to Cluster observations of a magnetic structure with a $B_{z}$ south-to-north (GSE, i.e. BBF-type) bipolar signature, accompanied by mostly monopolar variations in $B_{x}$ and $B_{y}$. It was encountered at $X_{\mathrm{GSE}} \approx-19 R_{E}$ on 22 August 2001 10:08:30 UT during a substorm expansion phase. For further discussion on the associated substorm see Lui et al. (2006). As noted, a different tailward propagating plasmoid observed around 09:50 UT during the same substorm is discussed by Lui et al. (2007). We will recover in detail the geometry, size and orientation and calculate magnetic fluxes and currents within the rope. We shall then compare our results with single-spacecraft methods, such as minimum variance analysis (Sonnerup and Cahill, 1967), force-free fitting (Lepping et al., 1990) and curlometer techniques (e.g. Robert et al., 1998). (The latter two methods have already been applied to this event by
Slavin et al. (2003b).) These questions are posed with an eye to future THEMIS applications: How robust are the results provided by these widely used methods? For the interpretation of THEMIS observations it is essential to be aware of the limitations of single-spacecraft compared to multispacecraft-methods, as the time evolution of the structures is essentially sampled in a single-spacecraft manner at various distances downtail by the spacecraft on this mission. In this respect it might also be expected that static models are increasingly unreliable the closer the flux rope is observed to the reconnection site because of a presumably more dynamic environment.

\section{Data}

On 22 August 2001 the Cluster mean position in the interval 10:08:00-10:09:00 UT was $[-18.8,-3.3,-1.0] R_{E}$ in GSE coordinates, with an average spacecraft separation of $1711 \mathrm{~km}$. In Fig. 1 an overview of magnetic field and plasma data is given, with data plotted at time resolutions of $0.0450 \mathrm{~s}$ and $4.021 \mathrm{~s}$, respectively. Displayed are the FGM (Balogh et al., 2001) and CIS (Rème et al., 2001) measurements of magnetic field and plasma parameters. For $\mathrm{C} 1$ and $\mathrm{C} 3$, we use data from CIS/HIA for ions without mass discrimination, and we also plot proton bulk velocities for $\mathrm{C} 4$ from CIS/CODIF. For C2 no plasma bulk parameters were available. The interval used for GS reconstruction is delimited by the two solid vertical lines. During this interval, the magnetic field components are quite similar at $\mathrm{C} 1, \mathrm{C} 3$ and $\mathrm{C} 4$, with a south-to-north bipolar variation in $B_{z}$ and a negative peak in $B_{x}$ and $B_{y}$, with a peak magnetic field strength $B_{\max , 134}=30.5-32.4 \mathrm{nT}$. As noted by Slavin et al. (2003b), the delay of the magnetic signatures and the plasma velocity imply that the rope is being convected with the flow. The configuration of the Cluster spacecraft in the GSE-YZ and $\mathrm{XY}$ planes is indicated in Fig. 3.

In slight contrast, at $\mathrm{C} 2$, displaced on the duskward side with respect to the others (Fig. 3, bottom panel), $B_{x}$ and $B_{z}$ exhibit a bipolar variation, the peak magnetic field strength is clearly lower $\left(B_{\max , 2}=25.8 \mathrm{nT}\right)$, and the profile is more flat. In summary, all four Cluster spacecraft encountered a rotation of the magnetic field vector qualitatively typical of a left-handed helical magnetic flux rope, with an axial field roughly pointing toward dawn $\left(B_{y}<0\right)$. According to Hughes and Sibeck (1987) this is consistent with the almost continuous negative $B_{y}(\mathrm{GSM})$ polarity of the interplanetary magnetic field (IMF) observed by ACE (not shown) from 08:35 to 09:45 UT. During the encounter, the ion $\beta$ measured at $\mathrm{C} 1$ and $\mathrm{C} 3$ drops from $\approx 1$ (typical of the central plasma sheet) to $\approx 0.2-0.3$.

At $X_{\mathrm{GSE}}=-19 R_{E}$, the earthward propagating flux rope might also be encountered rather close to the reconnection site which statistical studies estimate as $X_{\mathrm{GSE}} \approx-20 \pm 5 R_{E}$ (Miyashita et al., 2009, and references therein). Because of 
continuously enhanced solar wind energy input for $11 / 2 \mathrm{~h}$ prior to the encounter (on average IMF $B_{z} \approx-5 \mathrm{nT}$, solar wind velocity $V \approx 570 \mathrm{~km} / \mathrm{s}$ ) the reconnection site can be expected to be closer to Earth (Nagai et al., 2005).

\section{Application of methods and results}

\subsection{Single-spacecraft GS technique}

The single-spacecraft version of the Grad-Shafranov technique (Hau and Sonnerup, 1999; Hu and Sonnerup, 2002) (GS) is applied to the $\mathrm{C} 3$ spacecraft data acquired in the interval 10:08:33-10:08:44 UT (vertical lines in Fig. 1), because of complete coverage of the necessary magnetic field and plasma data (proton bulk velocity $\boldsymbol{V}$, ion number density $N_{i}$, ion temperature $T_{i}$, ion pressure $p=N_{i} k T_{i}$ ); the results for $\mathrm{C} 1$ are similar. A deHoffmann-Teller (HT) analysis (e.g. Khrabrov and Sonnerup, 1998) is applied to the data, which searches for the frame of reference where the convection electric field $\boldsymbol{E}=\left(\boldsymbol{V}-\boldsymbol{V}_{H T}\right) \times \boldsymbol{B} \approx 0$ and the flow is fieldaligned. Due to the short interval (12s) only 3 data points can be used, linearly interpolated to the magnetic field data resolution, yielding $\boldsymbol{V}_{H T}=[799.0,178.1,15.4] \mathrm{km} / \mathrm{s}(\mathrm{GSE})$, with a correlation coefficient between $\boldsymbol{V}_{H T} \times \boldsymbol{B}$ and $\boldsymbol{V} \times \boldsymbol{B}$ of $c c_{H T}=0.986$. While keeping in mind that the short duration and the few data points make $\boldsymbol{V}_{\boldsymbol{H} \boldsymbol{T}}$ rather uncertain, we note that $\boldsymbol{V}_{\boldsymbol{H} T}$ seems to have a significant cross tail component toward dusk besides the main component along GSE + X. An initial invariant axis is obtained through minimum variance analysis (MVA), yielding a flux rope axis as the intermediate variance direction of $\theta=36^{\circ}$ (the inclination to the ecliptic, from $-90^{\circ}$ to $+90^{\circ}$ ) and $\phi=237^{\circ}$ (measured from GSE $+\mathrm{X}\left(0^{\circ}\right)$ toward GSE $\left.+\mathrm{Y}\left(90^{\circ}\right)\right)$. The ratio of intermediateto-minimum eigenvalues was found to be $\lambda_{2} / \lambda_{3}=10$ indicating a robust determination (Lepping and Behannon, 1980). Interestingly, applying MVA using normalized unit vectors (MVU) gives $\left(\theta=3^{\circ}, \phi=257^{\circ}\right)$ with $\lambda_{2} / \lambda_{3}=3.4$.

The GS invariance direction $\hat{z}^{\prime}$ is then calculated by minimizing the scatter of the pressure

$$
P_{t}(A)=B_{z}^{2} / 2 \mu_{0}+p
$$

i.e. locating where $P_{t}(A)$ is closest to being singlevalued. Here $A(x, y)$ is the vector potential and the magnetic field is represented in the integration domain by $\mathbf{B}=\left[\partial A(x, y) / \partial y,-\partial A(x, y) / \partial x, B_{z}(x, y)\right]$ (Hau and Sonnerup, 1999). The result is $\left(\theta=42 \pm 5^{\circ}, \phi=259 \pm 9^{\circ}\right)$. (For a determination of the error bars see $\mathrm{Hu}$ et al., 2004). This inclination is considerably higher than the one obtained from MVU but quite similar to MVA. The reconstruction coordinate system (for details see $\mathrm{Hu}$ and Sonnerup, 2002) is: $\hat{\boldsymbol{x}}^{\prime}=[-0.976,-0.014,-0.216]$, $\hat{\boldsymbol{y}}^{\prime}=[0.167,-0.687,-0.708], \quad \hat{z}^{\prime}=[-0.138,-0.727,0.672]$ (the flux rope axis) in GSE.
We now obtain maps of the field lines. Only those field lines which are crossed twice by a spacecraft and for which $P_{t}(A)$ is single-valued can be reconstructed reliably. In Fig. 2a these are separated by a vertical line at $A_{b}=-0.009 \mathrm{~T} \mathrm{~m}$ from the rest of the observations. The function $P_{t}(A)$ is fitted by a polynomial with exponential tails in regions of the integration domain which are not covered by observations. The residue $R_{f}=0.06$ (Hu et al., 2004) is found to be low, indicating a good quality fit, and $P_{t}(A)$ is close to being single-valued. The Grad-Shafranov equation

$\frac{\partial^{2} A}{\partial x^{2}}+\frac{\partial^{2} A}{\partial y^{2}}=-\mu_{0} \frac{d P_{t}(A)}{d A}$

is then numerically solved to produce maps of $A(x, y)$ and $B_{z}(A(x, y)$,$) . This map is shown in Fig. 2c. The black con-$ tour lines represent isocontours of $A$ and thus give the field in the transverse $\left(\hat{\boldsymbol{x}}^{\prime}-\hat{\boldsymbol{y}}^{\prime}\right)$ plane. The strength of the longitudinal (toroidal) component of the field, $B_{z}$, is color-coded according to the color bar on the right. The magnetic field components by all 4 Cluster spacecraft are plotted as arrows projected into the reconstruction $\hat{\boldsymbol{x}}^{\prime}-\hat{\boldsymbol{y}}^{\prime}$ plane, i.e. the plane perpendicular to the invariance directions $\hat{z}^{\prime}$. Note that positive $\hat{\boldsymbol{y}}^{\prime}$ points south of the ecliptic, so that $\mathrm{C} 2$ (the lowest trajectory) is situated to the north of the ecliptic. For every s/c, the plotted arrows are time-shifted to $\mathrm{C} 3$ to include the effect that the rectangular integration domain moves with constant $V_{H T}$ over the Cluster tetrahedron (see Möstl et al., 2009).

The maximum separation along $\hat{\boldsymbol{y}}^{\prime}$ is between $\mathrm{C} 2$ and C4 $1754 \mathrm{~km}$, see Fig. 2c. For $\hat{\boldsymbol{x}}^{\prime}$, this maximum distance is between $\mathrm{C} 1$ and $\mathrm{C} 3-1715 \mathrm{~km}$, i.e. approximately antisunward. In $\hat{z}^{\prime}$ direction, $\mathrm{C} 4$ is $1904 \mathrm{~km}$ (pointing out of the $\hat{\boldsymbol{x}}^{\prime}-\hat{\boldsymbol{y}}^{\prime}$ plane) away from C3, see also top panel in Fig. 3 . We thus infer that the flux rope axis orientation remains at least the same for $1900 \mathrm{~km}$ because qualitatively the magnetic field rotation is similar at C3 and C4. C2 is $-1676 \mathrm{~km}$ away from $\mathrm{C} 3$ in $\hat{\boldsymbol{y}}^{\prime}$ and has a much smaller impact parameter (i.e. the minimum distance from the axis) than $\mathrm{C} 1, \mathrm{C} 3$ and $\mathrm{C} 4$. Comparing the map reconstructed from the $\mathrm{C} 3$ observations at the location of $\mathrm{C} 2$ with the actual observations by $\mathrm{C} 2$ shows that the assumption of $2 \frac{1}{2}$-dimensionality is approximately fulfilled and that CLUSTER encountered a magnetic configuration possessing an invariant axis, i.e. a flux rope. This will be further discussed in Sect. 3.4. It is intriguing that C2 measures a lower total field strength and slightly different field rotations (especially in the front part) than the other three spacecraft while single-spacecraft GS predicts that C2 should have a very low impact parameter, i.e. that $\mathrm{C} 2$ should cross the flux rope very close to its axis. Further, the lower total field strength at $\mathrm{C} 2$ is opposite to what is expected from the classic force-free (FF) model.

\subsection{Multi-spacecraft GS technique}

The multi-spacecraft extension to the GS technique (Hasegawa et al., 2005), which we call GS2, is applied to the 
(a)

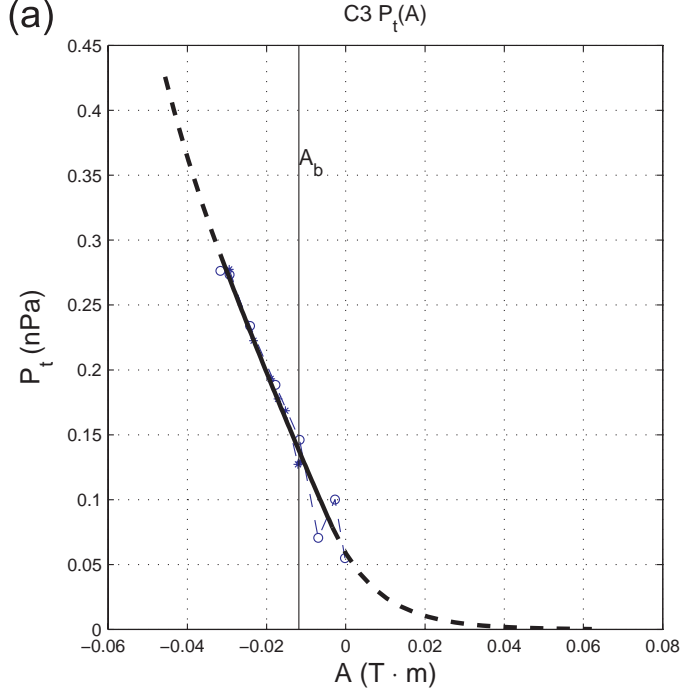

(c)

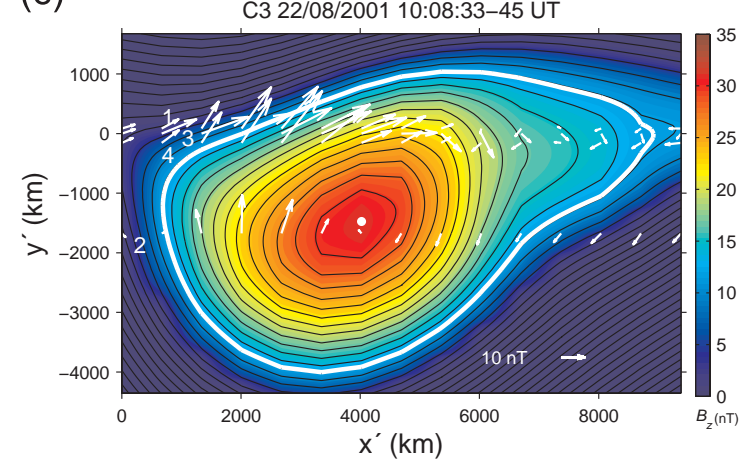

(b)

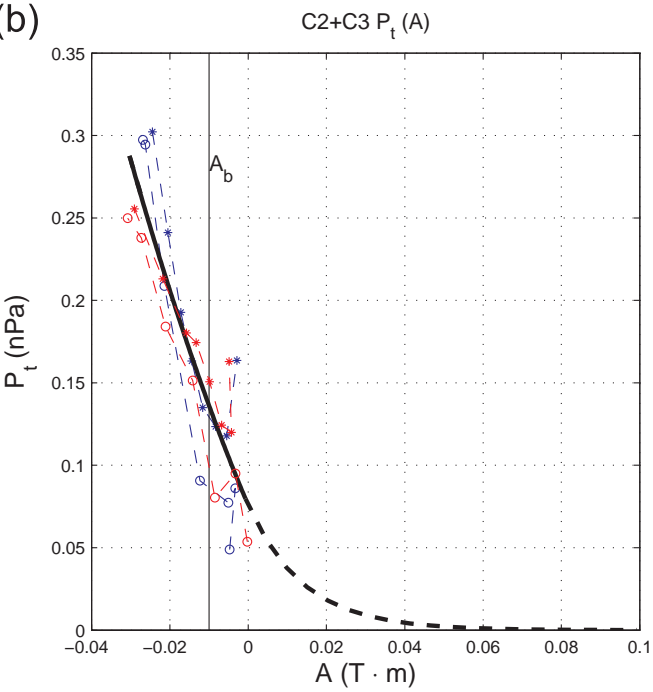

(d)

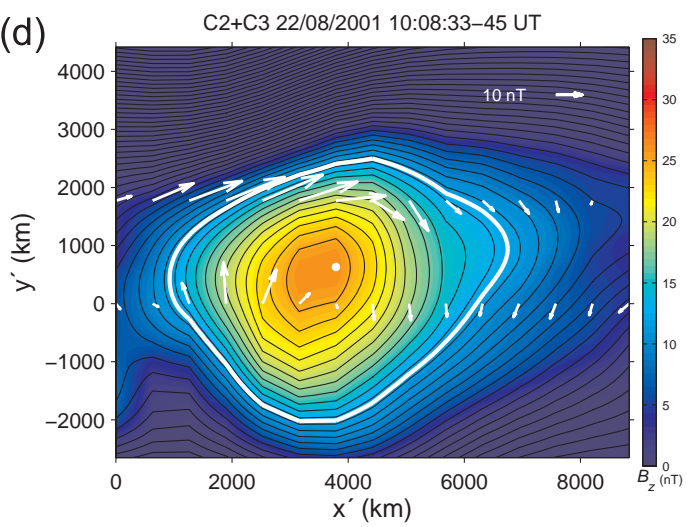

Fig. 2. (a) Single-s/c GS: $P_{t}(A)$ plot for $\mathrm{C} 3$ with the 2 nd order polynomial fitting function (solid black) with exponential tails (dashed black) used for the right hand side of the GS Eq. (2). Circles and stars represent inbound and outbound measurements, respectively. (b) Multi-s/c GS2: Combined $P_{t}(A)$ plot (red C3, blue C2). (c) GS: Magnetic field map integrated from C3 observations taken as initial values. $B_{z}$ is color coded, pointing out of the paper, and maximizes at the white dot. Black contours are magnetic field lines in the paper plane, the white contour corresponds to the vertical line $\left(A_{b}\right)$ in Fig. 2a. Arrows indicate observations along each spacecraft trajectory. From top to bottom: C1-C3-C4-C2. The structure propagates toward Earth to the left, positive $\hat{\boldsymbol{y}}^{\prime}$ points south of the ecliptic (see also Fig. 3 ). Grid size: $15 \times 91$. (d) GS2: Combined magnetic field map from C2 and C3. The arrows are again magnetic field components in the $\hat{\boldsymbol{x}}^{\prime}-\hat{\boldsymbol{y}}^{\prime}$ (paper) plane along the trajectories of $\mathrm{C} 3$ (upper) and C2 (lower). Also note the same scale for the color coded $B_{z}$ as in Fig. $2 \mathrm{c}$. Grid size: $15 \times 113$.

$\mathrm{C} 2$ and $\mathrm{C} 3$ measurements. As $\mathrm{C} 1$ and $\mathrm{C} 4$ are very close to $\mathrm{C} 3$ in the $\hat{\boldsymbol{y}}^{\prime}$-direction and reconstruction from $\mathrm{C} 1$ (not shown) is practically identical to the one from $\mathrm{C} 3$, we calculate a combined magnetic field map, ingesting the observations from $\mathrm{C} 2$ and $\mathrm{C} 3$. Using the most widely separated spacecraft in the $\hat{\boldsymbol{x}}^{\prime}-\hat{\boldsymbol{y}}^{\prime}$ plane is the best way to obtain the most complete view of the flux rope cross section with the data to hand. Because plasma observations are not available from $\mathrm{C} 2$, we estimate the plasma pressure at $\mathrm{C} 2$ with the method introduced by Hasegawa et al. (2005) using electron density measurements from the EFW instrument (Pedersen et al., 2001). A combined deHoffmann-Teller analysis for C1 and C3 (now with 6 data points) leads to $\mathbf{V}_{H T}=[668.4,143,-43.7] \mathrm{km} / \mathrm{s}$
(GSE), with a somewhat lower $c c_{H T}=0.9576$. For the subsequent analysis the data interval on $\mathrm{C} 2$ was time-shifted again to $\mathrm{C} 3$ because the rectangular integration box moves with constant $\boldsymbol{V}_{H T}$ from $\mathrm{C} 3$ to $\mathrm{C} 2$.

Figure $2 \mathrm{~b}$ shows a combined $P_{t}(A)$ plot fitted with a 2 nd order polynomial (solid line), with a fitting residue $R_{f}=0.12$. From this a combined magnetic field map was created from the individual field maps for C2 and C3 according to Sonnerup et al. (2004), with a Gaussian window function width of $25 \%$ of the reconstruction domain. By optimizing the correlation coefficients of predicted magnetic field components by the combined map with the actual observations to $c c=0.91$ we find an invariant axis orientation $\theta=40^{\circ}$ 

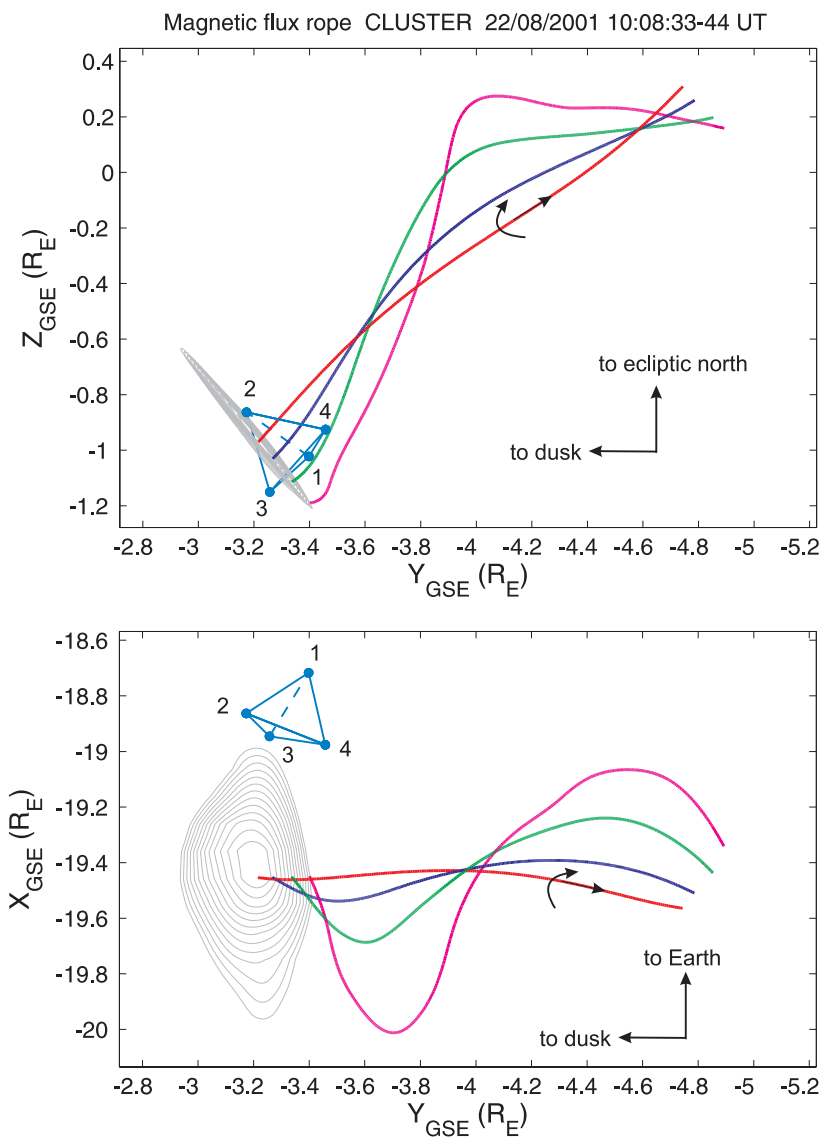

Fig. 3. Combined magnetic field map from $\mathrm{C} 2$ and $\mathrm{C} 3$ (Fig. 2d), drawn as left-handed spiral field lines in 3-D and placed in a GSE coordinate system. Top: View in the GSE YZ-plane looking toward Earth. The positions of the Cluster tetrahedron is indicated with $\mathrm{C} 1$ being the spacecraft displaced most Earthward, i.e. in the plane of the paper. Along the red (almost straight) field line the axial field direction is indicated and how the other three field lines spiral around the axis in a left-handed sense. Bottom: The same as above seen in the GSE XY-plane looking toward ecliptic South.

and $\phi=265^{\circ}$, in GSE, with $\hat{z}=[-0.0668,-0.7631,0.6428]$ (GSE), which is quite similar to the one inferred from GS. Figure $2 \mathrm{~d}$ shows the integrated magnetic field map in a plane perpendicular to $\hat{z}^{\prime}$. Now the rope is more extended in $-\hat{\boldsymbol{y}}^{\prime}$ and has an aspect ratio of about 1:1.5, elongated approximately along the direction of propagation. It resembles a "raindrop" shape, i.e. flattened in front and elongated at the rear, though this is less here than in Fig. 2c. The higher residue than for the one computed with single-s/c GS and the lower correlation compared to another event $c c$ (c.f. Hasegawa et al., 2007) indicates that the combined observations by $\mathrm{C} 2$ and $\mathrm{C} 3$ are deviating somewhat from the GS model assumptions.

\subsection{Field line turns per $R_{E}$; force-free test}

Figure 3 shows 3-D views of the flux rope's spiral magnetic field lines placed in the GSE coordinate system, with the Cluster tetrahedron indicated. To obtain this, we assumed invariance for $2 R_{E}$ and stacked 20 maps similar to that of Fig. $2 \mathrm{~d}$ along $\hat{z}^{\prime}$. In the resulting data cube four field lines were traced, from the axis (red, almost straight field line) to the outer boundary (pink field line, equivalent to $A_{b}$ ). The number of turns $n$ these four field lines make is $n=0.46-0.5$ per $R_{E}$. Thus the flux rope has field lines twisted by approximately the same amount irrespective of radial distance from the axis. For a length $L \approx 1.5-3 R_{E}$ along $\hat{z}^{\prime}$, the typical scale size of BBF's (Nakamura et al., 2004), the full number of turns, $N$, is $0.69-1.5$ and thus the flux rope is composed of only weakly wound field lines but could still eventually go kink unstable (e.g. Hood and Priest, 1981; McClymont and Craig, 1987). The close-to-uniform twist is again at variance with the constant- $\alpha$ force-free model but resembles another type of flux rope, namely, a nonlinear $(\alpha \neq$ const $)$ but still force-free "Gold-Hoyle" tube (e.g. Priest, 1982), such as has been reported in the solar wind (Farrugia et al., 1999). However, below we will argue that a significant part of the flux rope under study here is not force-free.

To investigate the latter, we also looked where in the combined magnetic field map (Fig. 2d) $\boldsymbol{J} \| \boldsymbol{B}$. To this end, Fig. 4 shows the ratio of the perpendicular to the parallel currents $\varrho=\left|J_{\perp}\right| /\left|J_{\|}\right|$for the combined magnetic field map. While the core of the rope is force-free, arbitrarily defined as $\varrho<0.3$ (inside the white dashed contour), and has an approximately round shape, the boundaries and in particular the elongated back part is dominated by perpendicular currents. About $1 / 3$ of the area inside the boundary $A_{b}$ (the white solid contour) is not force-free $(\varrho>0.3)$. With this we intend only to show a general qualitative trend: to be able to make a strong conclusion about the detailed force-free state of the flux rope, a knowledge of the small-scale features of $\boldsymbol{J}$ and $\boldsymbol{B}$ would be necessary, while the GS technique is designed to recover a smoothed image (in $2 \frac{1}{2} \mathrm{D}$ ) of a given magnetic structure.

\subsection{Further support for the flux rope interpretation}

Recently, 3-D MHD simulations of tail reconnection by Shirataka et al. (2006) have revealed that similar signatures in magnetic field, plasma density and temperature usually interpreted as helical magnetic flux ropes can also be produced by 3-D guide field reconnection jets at a single $\mathrm{X}$ line. No helical field lines exist in this simulation. Nevertheless, Hasegawa et al. (2007) have reconstructed a helical flux rope from these simulated data with the GS method, concluding that it is impossible to deduce the magnetic topology from the GS maps alone. Hasegawa et al. (2007) have presented guidelines in interpretation of the GS results in order to discriminate between flux ropes and reconnection jets, and applying these, we find further support for the flux rope 
Table 1. Results of the multi-spacecraft Grad-Shafranov reconstruction (GS2) for the CLUSTER flux rope on 22 August 2001 10:08:33-10:08:45 UT. Minimum variance analysis (MVA) and single s/c GS was carried out for $\mathrm{C} 3$, and force-free fitting (FF) results were obtained by Slavin et al. (2003b). For comparison, the deHoffmann-Teller analysis was done independently for C1, C3 as well for the combined set $(\mathrm{C} 1+\mathrm{C} 3)$. Further, $H$ the handedness, $D$ is the diameter, $B_{z}$ the central axial field strength, $j_{z}$ the central current density, $J$ the full axial current, $\Phi_{t}$ the axial flux and $\Phi_{p}$ the poloidal flux for $L=1.5-3 R_{E} . J, \Phi_{t}$ and $\Phi_{p}$ were integrated over the area inside the white contour $\left(<A_{b}\right)$ in Fig. $2 \mathrm{~d}$.

\begin{tabular}{cc}
\hline start, UT & $10: 08: 33$ \\
duration, s & 11 \\
$\mathrm{C} 1: \mathbf{V}_{H T}, \mathrm{~km} / \mathrm{s}$ & $(571.6,94.9,-143.0)$ \\
$\mathrm{C} 3: \mathbf{V}_{H T}, \mathrm{~km} / \mathrm{s}$ & $(799.0,178.1,15.4)$ \\
$\mathrm{C} 1+\mathrm{C} 3: \mathbf{V}_{H T}, \mathrm{~km} / \mathrm{s}$ & $(668.4,143,-43.7)$ \\
$\theta$, deg $(\mathrm{GS}, \mathrm{FF}, \mathrm{MVA})$ & $40(42 \pm 5,-12$ to 17,36$)$ \\
$\phi$, deg $(\mathrm{GS}, \mathrm{FF}, \mathrm{MVA})$ & $265(259 \pm 9,200$ to 270,237$)$ \\
$\mathrm{H}(\mathrm{GS}, \mathrm{FF})$ & $\mathrm{L}(\mathrm{L}, \mathrm{L})$ \\
$\mathrm{D}, R_{E}(\mathrm{FF})$ & $1(0.96-1.04)$ \\
$B_{z}, \mathrm{nT}(\mathrm{GS}, \mathrm{FF})$ & $26.4(31.7,28.9-38.9)$ \\
$j_{z}, \mathrm{nA} / \mathrm{m}^{2}(\mathrm{GS}, \mathrm{FF})$ & $9(12,15-19)$ \\
$J,(\mathrm{GS}) \mathrm{MA}$ & $0.156(0.144)$ \\
$\Phi_{t}, 10^{5} \mathrm{~Wb}$ & 1.9 \\
$\Phi_{p}, 10^{5} \mathrm{~Wb}$ & $2.25-4.5$ \\
\hline
\end{tabular}

interpretation reached above. The following features support the flux rope interpretation: (1) The plasma pressure is not enhanced in the front part of the tube (see the profiles for $N_{i}$ and $T_{i}$ in Fig. 1), (2) the independent deHoffmann-Teller velocities derived from $\mathrm{C} 1$ and $\mathrm{C} 3$ are roughly consistent in direction and magnitude (see Table 1), (3) transverse magnetic field lines (in the $\hat{\boldsymbol{x}}^{\prime}-\hat{\boldsymbol{y}}^{\prime}$ plane) appear elongated in the direction of motion (Fig. 2c and d), and (4) the functions $P_{t}(A)$ (Fig. 2a, b) and $B_{z}(A)$ (not shown) appear approximately as single-valued.

\subsection{Comparison with other methods}

We now compare the results of various techniques applied to the same flux rope. Table 1 shows the results from the methods of (1) minimum variance analysis (MVA) applied to the C3 measurements, (2) force-free fitting to every spacecraft individually by Slavin et al. (2003b), (3) single-spacecraft GS to C3 and (4) GS2 applied to the combined set of C2 and C3 observations.

It follows from Table 1 that there is some agreement on basic parameters such as handedness and size. The biggest differences are in the inclination (MVA, GS, GS2: $\approx 40^{\circ}(\mathrm{GSE})$; FF and MVU: $\approx 0^{\circ}(\mathrm{GSE})$ ). The $\approx 40^{\circ}$ inclination in GSE corresponds to $\approx 10^{\circ}$ in GSM, so that the flux rope axis lies almost in the GSM equatorial plane. Due to the difference in axis orientation the impact parameters from force-free fitting also differ - Slavin et al. (2003b) found C1, C2 and C4 to

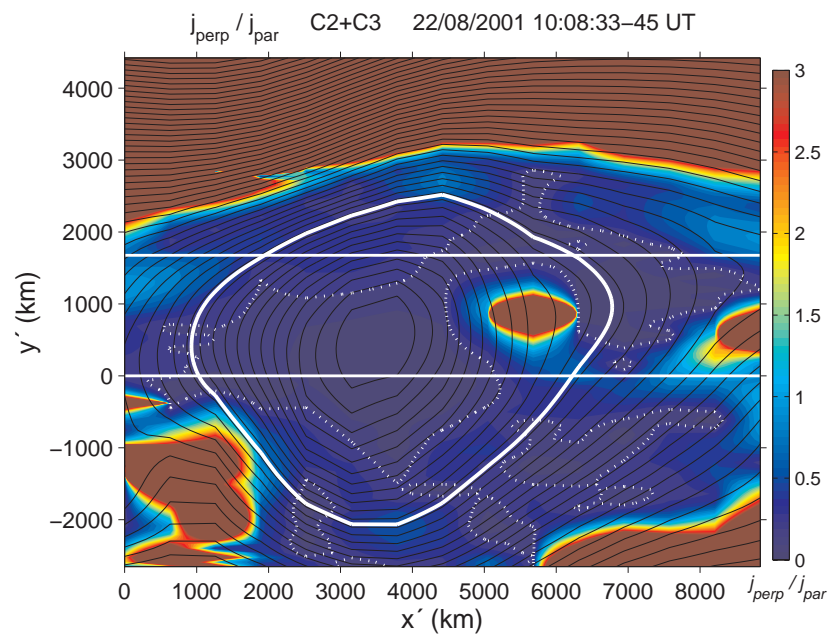

Fig. 4. The ratio of the perpendicular to the parallel currents $\varrho=\left|\boldsymbol{J}_{\perp}\right| /\left|\boldsymbol{J}_{\|}\right|$(color coded), showing the force-free core of the flux rope where $r \approx 0$. The dotted white contour indicates the level where $\varrho=0.3$. Solid black contours are field lines in the $\hat{\boldsymbol{x}}^{\prime}-\hat{\boldsymbol{y}}^{\prime}$ plane. The solid white contour corresponds to the boundary $A_{b}$, and white horizontal lines are trajectories (upper $\mathrm{C} 3$, lower $\mathrm{C} 2$ ).

pass north (i.e. in the direction of $+Z_{\mathrm{GSM}}$ ) and $\mathrm{C} 3$ south of the axis, whereas the GS maps show that $\mathrm{C} 2$ crosses north of the axis at a different impact parameter and C1, C3 and $\mathrm{C} 4$ cross south of the axis. This is more consistent with the magnetic field observations (Fig. 1; note that MVA, MVU and curlometer do not yield impact parameters). Also the axial field strength $B_{z}$ deduced by GS2 is lower by one-third and the central axial current density $j_{z}$ is roughly half the values inferred from FF. Further, single-s/c GS seems to overestimate $B_{z}$ and $j_{z}$ if the distance from the axis turns out to be large. Additionally the combined magnetic field map is more extended perpendicular to the direction of motion than the single-s/c map. We think that the discrepancies between FF and GS, GS2 likely stem from the non-force-free treatment by the GS technique, which deduces the orientation not only from the magnetic field profile but includes plasma pressure in its determination as well. Thus single-spacecraft techniques should be used with these caveats in mind when applied to THEMIS data, where an event is seen successively at individual spacecraft, located in a "pearl" configuration.

Additionally, by considering curlometer results for the current density perpendicular to local magnetic field, Slavin et al. (2003b) have found deviations from the force-free state which were stronger in the front part than in the rear part. Indeed we can confirm this statement by looking at the trajectory of $\mathrm{C} 3$ (close to $\mathrm{C} 1$ and $\mathrm{C} 4$ ) in Fig. 4, which shows a force-free back and non-force-free front part; only for $\mathrm{C} 2$ is it the reverse. Slavin et al. (2003b) attributed these deviations from the force-free state to the fact that the structure runs into denser plasma as it propagates earthwards. This compression 
causes an asymmetry in the magnetic field profile such that the transverse magnetic field $\left(B_{x}^{\prime}\right.$ and $B_{y}^{\prime}$ ) is stronger in the front part than in the back. Such an effect is evident especially in the $\mathrm{C} 2$ magnetic field observations made closer to the rope's core (the lowest trajectory in Fig. 2c and d). The same asymmetry is also seen in the field maps generated by Hasegawa et al. (2007) and Zhang et al. (2007) for two other events.

A reason for the non-force-free state could be that the flux rope was encountered in a very early stage in its evolution and it has not yet had time to relax to a minimum energy (force-free) configuration. It can thus be expected in this case that the non-force-free curlometer and GS techniques yield more accurate results, with the drawback that curlometer does not yield impact parameters and a map of the crosssection compared to the GS techniques.

\section{Summary and conclusions}

In this paper we reconstructed a magnetic structure observed by Cluster at $X_{\mathrm{GSE}}=-19 R_{E}$ during the expansion phase of a substorm. Its internal structure was composed of helical magnetic field lines, with significant deviations from the Lundquist linear force-free model. The field lines were estimated to wind around the axis with about half a turn per $R_{E}$. Spacecraft C2, crossing closer to the flux rope center than the other three spacecraft, observed a lower total magnetic field strength closer to the core, which is at variance with analytical models in current use. We also found one third of its cross section to be non-force-free: While the core was approximately force-free, the boundary regions were not. In using the GS methods and minimum variance analysis we infer that the flux rope originated from an only slightly tilted current sheet $\left(+10^{\circ}\right.$ to the GSM equatorial plane), whereas minimum variance analysis using normalized vectors and force-free fitting yielded a stronger inclination $\left(-30^{\circ}\right)$. The orientation of the current sheet tilt $\left(+10^{\circ}\right)$ towards dusk, such that the duskside part of the northern lobe is below the geomagnetic equator, is consistent with observed negative IMF $B_{y}$ and the left-handed chirality of the flux rope. The flux rope carries earthward a total axial current of 0.16 MA which is directed opposite to the axial field since the flux rope is left-handed. In summary, the rope possesses a helical structure, consistent with its having originated from multiple $\mathrm{X}$-line reconnection (Slavin et al., 2003a,b). It also became clear during this study that this event turns out not to be one of the best suited for GS reconstruction: There are only a few plasma data points due to the high velocity, which results in a very short event duration, the pressure at $\mathrm{C} 2$ had to be estimated, and dynamic effects are not insignificant. However, this illustrates the application of the static technique facing these problems. We have still found a good qualitative agreement between the reconstruction and multi-spacecraft observations and we think that the derived flux rope parameters are credible.
Accordingly, we speculate that, due to the enhanced solar wind energy input and the encounter at $X_{\mathrm{GSE}}=-19 R_{E}$, an observation quite close to the reconnection site is likely; this is also supported by its high velocity $(\approx 600-700 \mathrm{~km} / \mathrm{s})$ and strong axial field strength $(>25 \mathrm{nT})$ in the context of other events studied by Hasegawa et al. (2007) and Zhang et al. (2007). In this case non-force-free techniques are to be preferred, even though they can yield some error for higher impact parameters if used solely in single-spacecraft fashion. The single-spacecraft GS method overestimated the axial field strength and axial current by roughly $25 \%$ compared to the multi-spacecraft technique (GS2). Thus we corroborate the conclusions of Slavin et al. (2003b) who noted shortcomings of single-spacecraft methods and violation of the force-free condition. To this we have added essential elements and techniques for the analysis of magnetotail flux ropes, which hold promise for future work.

It is interesting to note that the flux ropes' poloidal flux per unit length, $\approx 0.02 \mathrm{~T} \mathrm{~m}$, is in the same order as the reconnected flux inferred from a time-dependent Petschek-type reconnection model (e.g. Biernat et al., 1987; Semenov et al., 2005; Ivanova et al., 2007; Kiehas et al., 2008) for different events. From this we conclude that approaches quite distinct from one another (see Fig. 1 in Semenov et al., 2005) may complement each other in future studies to infer properties of reconnection in the magnetotail.

An earlier flux-rope traveling tailward during the same substorm expansion phase (around 09:50 UT) was extensively discussed by Lui et al. (2007). They discovered an irregular magnetic field structure in its inner core which indicated significant deviations from the expected helical nature of magnetic field lines in our usual ideas of a flux rope. In particular they found that the electric field and current density directions reversed in going from the leading to the trailing edge. Its size was estimated as $\approx 2 R_{E}$ and the total current as $0.8 \mathrm{MA}$. They evaluated the Lorentz-force and found it to be non-zero, implying also that the rope was not forcefree. These results, derived for a different flux rope and using different techniques (e.g. curlometer) have many features which we reproduce in a different form and using a different methodology. The two examples together indicate that the features first shown by Lui et al. (2007) and derived here also may be quite common in magnetotail flux-ropes. This is an issue worth pursuing.

Acknowledgements. We thank the instrument teams and the Cluster Active Archive for making the data easily available. C.M. is supported by the young researchers fund of the Steiermärkische Sparkasse. We acknowledge the Austrian Fonds zur Förderung der wissenschaftlichen Forschung for support under projects P20145N16 and P20314-N16. This work is supported by NASA Cluster grants to UNH and by NASA grant NNG06GD41G and NNX08D11G.

Editor in Chief W. Kofman thanks T. Nagai for his help in evaluating this paper. 


\section{References}

Angelopoulos, V.: The THEMIS Mission, Space Sci. Rev., 141, 534, doi:10.1007/s11214-008-9336-1, 2008.

Balogh, A., Carr, C. M., Acua, M. H., Dunlop, M. W., Beek, T. J., Brown, P., Fornacon, K.-H., Georgescu, E., Glassmeier, K.H., Harris, J., Musmann, G., Oddy, T., and Schwingenschuh, K.: The Cluster Magnetic Field Investigation: overview of in-flight performance and initial results, Ann. Geophys., 19, 1207-1217, 2001, http://www.ann-geophys.net/19/1207/2001/.

Biernat, H. K., Heyn, M. F., and Semenov, V. S.: Unsteady Petschek reconnection, J. Geophys. Res., 92, 3392-3396, 1987.

Burlaga, L. F.: Magnetic clouds and force-free fields with constant alpha, J. Geophys. Res., 93, 7217-7224, doi:10.1029/ JA093iA07p07217, 1988.

Farrugia, C. J., Janoo, L. A., Torbert, R. B., Quinn, J. M., Ogilvie, K. W., Lepping, R. P., Fitzenreiter, R. J., Steinberg, J. T., Lazarus, A. J., Lin, R. P., Larson, D., Dasso, S., Gratton, F. T., Lin, Y., and Berdichevsky, D.: A Uniform-Twist Magnetic Flux Rope in the Solar Wind, in: American Institute of Physics Conference Series, edited by: Suess, S. T., Gary, G. A., and Nerney, S. F., vol. 471 of American Institute of Physics Conference Series, p. 745, 1999.

Hasegawa, H., Sonnerup, B. U. Ö., Klecker, B., Paschmann, G., Dunlop, M. W., and Rème, H.: Optimal reconstruction of magnetopause structures from Cluster data, Ann. Geophys., 23, 973982, 2005, http://www.ann-geophys.net/23/973/2005/.

Hasegawa, H., Sonnerup, B. U. Ö., Owen, C. J., Klecker, B., Paschmann, G., Balogh, A., and Rème, H.: The structure of flux transfer events recovered from Cluster data, Ann. Geophys., 24, 603-618, 2006, http://www.ann-geophys.net/24/603/2006/.

Hasegawa, H., Nakamura, R., Fujimoto, M., Sergeev, V. A., Lucek, E. A., Rème, H., and Khotyaintsev, Y.: Reconstruction of a bipolar magnetic signature in an earthward jet in the tail: Flux rope or 3D guide-field reconnection?, J. Geophys. Res. (Space Physics), 112, 11206, doi:10.1029/2007JA012492, 2007.

Hau, L.-N. and Sonnerup, B. U. Ö.: Two-dimensional coherent structures in the magnetopause: Recovery of static equilibria from single-spacecraft data, J. Geophys. Res., 104, 6899-6918, doi:10.1029/1999JA900002, 1999.

Henderson, P. D., Owen, C. J., Alexeev, I. V., Slavin, J., Fazakerley, A. N., Lucek, E., and Rème, H.: Cluster observations of flux rope structures in the near-tail, Ann. Geophys., 24, 651-666, 2006, http://www.ann-geophys.net/24/651/2006/.

Hood, A. W. and Priest, E. R.: Critical conditions for magnetic instabilities in force-free coronal loops, Geophysical and Astrophysical Fluid Dynamics, 17, 297-318, 1981.

$\mathrm{Hu}$, Q. and Sonnerup, B. U. Ö.: Reconstruction of magnetic clouds in the solar wind: Orientations and configurations, J. Geophys. Res. (Space Physics), 107, 1142, doi:10.1029/2001JA000293, 2002.

Hu, Q., Smith, C. W., Ness, N. F., and Skoug, R. M.: Multiple flux rope magnetic ejecta in the solar wind, J. Geophys. Res. (Space Physics), 109, 3102, doi:10.1029/2003JA010101, 2004.

Hu, Q., Smith, C. W., Ness, N. F., and Skoug, R. M.: On the magnetic topology of October/November 2003 events, J. Geophys. Res. (Space Physics), 110, 9, doi:10.1029/2004JA010886, 2005.

Hughes, W. J. and Sibeck, D. G.: On the 3-dimensional structure of plasmoids, Geophys. Res. Lett., 14, 636-639, 1987.

Ivanova, V. V., Semenov, V. S., Penz, T., Ivanov, I. B., Sergeev, V. A., Heyn, M. F., Farrugia, C. J., Biernat, H. K., Nakamura, R., and Baumjohann, W.: Reconstruction of the reconnection rate from Cluster measurements: Method improvements, J. Geophys. Res. (Space Physics), 112, 10226, doi:10.1029/2006JA012183, 2007.

Khrabrov, A. V. and Sonnerup, B. U. Ö.: deHoffmann-Teller analysis, in: Analysis Methods for Multi-Spacecraft Data, edited by: Paschmann, G. and Daly, P. W., ESA SR-001; Noordwijk: ESA, p. 221, 1998.

Kiehas, S. A., Semenov, V. S., Kubyshkin, I. V., Kubyshkina, M. V., Penz, T., Biernat, H. K., and Nakamura, R.: Determination of reconnected flux via remote sensing, Adv. Space Res., 41, 12921297, doi:10.1016/j.asr.2007.05.069, 2008.

Lee, L. C.: A Review of Magnetic Reconnection: MHD Models, Physics of the Magnetopause, p. 139, 1995.

Lepping, R. P. and Behannon, K. W.: Magnetic field directional discontinuities. I - Minimum variance errors, J. Geophys. Res., 85, 4695-4703, doi:10.1029/JA085iA09p04695, 1980.

Lepping, R. P., Burlaga, L. F., and Jones, J. A.: Magnetic field structure of interplanetary magnetic clouds at 1 AU, J. Geophys. Res., 95, 11957-11965, 1990.

Lui, A. T. Y., Zheng, Y., Zhang, Y., Livi, S., Rème, H., Dunlop, M. W., Gustafsson, G., Mende, S. B., Mouikis, C., and Kistler, L. M.: Cluster observation of plasma flow reversal in the magnetotail during a substorm, Ann. Geophys., 24, 2005-2013, 2006, http://www.ann-geophys.net/24/2005/2006/.

Lui, A. T. Y., Dunlop, M. W., Rème, H., Kistler, L. M., Gustafsson, G., and Zong, Q.-G.: Internal structure of a magnetic flux rope from Cluster observations, Geophys. Res. Lett., 34, 7102, doi: 10.1029/2007GL029263, 2007.

Lundquist, S.: Magnetohydrostatic fields, Ark. Fys. 2, 361-365, 1950.

McClymont, A. N. and Craig, I. J. D.: The structure and stability of coronal magnetic fields, Sol. Phys., 113, 131-134, 1987.

Miyashita, Y., Machida, S., and Kamide, Y., et al.: A state-of-theart picture of substorm-associated evolution of the near-Earth magnetotail obtained from superposed epoch analysis, J. Geophys. Res., 114, A01211, doi:10.1029/2008JA013225, 2009.

Möstl, C., Miklenic, C., Farrugia, C. J., Temmer, M., Veronig, A., Galvin, A. B., Vršnak, B., and Biernat, H. K.: Two-spacecraft reconstruction of a magnetic cloud and comparison to its solar source, Ann. Geophys., 26, 3139-3152, 2008, http://www.ann-geophys.net/26/3139/2008/.

Möstl, C., Farrugia, C. J., Miklenic, C., Temmer, M., , Galvin, A. B., Luhmann, J. G., Kilpua, E. K. J., Leitner, M., NievesChinchilla, T., Veronig, A., and Biernat, H. K.: Multi-spacecraft recovery of a magnetic cloud and its origin from magnetic reconnection on the Sun, J. Geophys. Res. (Space Physics), 114, A04102, doi:10.1029/2008JA013657, 2009.

Nagai, T., Fujimoto, M., Nakamura, R., Baumjohann, W., Ieda, A., Shinohara, I., Machida, S., Saito, Y., and Mukai, T.: Solar wind control of the radial distance of the magnetic reconnection site in the magnetotail, J. Geophys. Res. (Space Physics), 110, 9208, doi:10.1029/2005JA011207, 2005.

Nakamura, R., Baumjohann, W., Mouikis, C., Kistler, L. M., Runov, A., Volwerk, M., Asano, Y., Vörös, Z., Zhang, T. L., Klecker, B., Rème, H., and Balogh, A.: Spatial scale of highspeed flows in the plasma sheet observed by Cluster, Geophys. Res. Lett., 31, 9804, doi:10.1029/2004GL019558, 2004.

Pedersen, A., Décréau, P., Escoubet, C.-P., Gustafsson, G., Laakso, 
H., Lindqvist, P.-A., Lybekk, B., Masson, A., Mozer, F., and Vaivads, A.: Four-point high time resolution information on electron densities by the electric field experiments (EFW) on Cluster, Ann. Geophys., 19, 1483-1489, 2001, http://www.ann-geophys.net/19/1483/2001/.

Priest, E. R.: Solar magneto-hydrodynamics, Dordrecht, Holland; Boston: D. Reidel Pub. Co., Hingham, 1982.

Rème, H., Aoustin, C., Bosqued, J. M., Dandouras, I., Lavraud, B., Sauvaud, J. A., Barthe, A., Bouyssou, J., Camus, Th., CoeurJoly, O., Cros, A., Cuvilo, J., Ducay, F., Garbarowitz, Y., Medale, J. L., et al.: First multispacecraft ion measurements in and near the Earths magnetosphere with the identical Cluster ion spectrometry (CIS) experiment, Ann. Geophys., 19, 1303-1354, 2001, http://www.ann-geophys.net/19/1303/2001/.

Robert, P., Dunlop, M. W., Roux, A., and Chanteur, G.: Accuracy of current density determination, in Analysis Methods for MultiSpacecraft Data, ed. G. Paschmann and P. W. Daly, ESA SR-001; Noordwijk: ESA), p. 395, 1998.

Schindler, K.: A theory of the substorm mechanism., J. Geophys. Res., 79, 2803-2810, 1974.

Semenov, V. S., Penz, T., Ivanova, V. V., Sergeev, V. A., Biernat, H. K., Nakamura, R., Heyn, M. F., Kubyshkin, I. V., and Ivanov, I. B.: Reconstruction of the reconnection rate from Cluster measurements: First results, J. Geophys. Res. (Space Physics), 110, 11217, doi:10.1029/2005JA011181, 2005.

Shirataka, N., Fujimoto, M., Hasegawa, H., and TanDokoro, R.: Reproducing the bipolar magnetic signature at the jet leading edge by three-dimensional reconnection with nonzero guide field, J. Geophys. Res. (Space Physics), 111, 7201, doi:10.1029/ 2005JA011521, 2006.

Slavin, J. A., Lepping, R. P., Gjerloev, J., Fairfield, D. H., Hesse, M., Owen, C. J., Moldwin, M. B., Nagai, T., Ieda, A., and Mukai, T.: Geotail observations of magnetic flux ropes in the plasma sheet, J. Geophys. Res. (Space Physics), 108, 1015, doi:10.1029/ 2002JA009557, 2003a.

Slavin, J. A., Lepping, R. P., Gjerloev, J., Goldstein, M. L., Fairfield, D. H., Acuna, M. H., Balogh, A., Dunlop, M., Kivelson, M. G., Khurana, K., Fazakerley, A., Owen, C. J., Reme, H., and Bosqued, J. M.: Cluster electric current density measurements within a magnetic flux rope in the plasma sheet, Geophys. Res. Lett., 30, 14-1, 2003b.
Snekvik, K., Haaland, S., Østgaard, N., Hasegawa, H., Nakamura, R., Takada, T., Juusola, L., Amm, O., Pitout, F., Rème, H., Klecker, B., and Lucek, E. A.: Cluster observations of a field aligned current at the dawn flank of a bursty bulk flow, Ann. Geophys., 25, 1405-1415, 2007,

http://www.ann-geophys.net/25/1405/2007/.

Sonnerup, B. U. O. and Cahill, Jr., L. J.: Magnetopause Structure and Attitude from Explorer 12 Observations, J. Geophys. Res., 72, 171-183, 1967.

Sonnerup, B. U. Ö. and Teh, W.-L.: Reconstruction of twodimensional coherent MHD structures in a space plasma: The theory, J. Geophys. Res. (Space Physics), 113, 5202, doi:10. 1029/2007JA012718, 2008.

Sonnerup, B. U. Ö., Hasegawa, H., and Paschmann, G.: Anatomy of a flux transfer event seen by Cluster, Geophys. Res. Lett., 31, 11803, doi:10.1029/2004GL020134, 2004.

Sonnerup, B. U. Ö., Hasegawa, H., Teh, W.-L., and Hau, L.-N.: Grad-Shafranov reconstruction: An overview, J. Geophys. Res. (Space Physics), 111, 9204, doi:10.1029/2006JA011717, 2006.

Walsh, A. P., Fazakerley, A. N., Wilson, R. J., Alexeev, I. V., Henderson, P. D., Owen, C. J., Lucek, E., Carr, C., and Dandouras, I.: Near-simultaneous magnetotail flux rope observations with Cluster and Double Star, Ann. Geophys., 25, 1887-1897, 2007, http://www.ann-geophys.net/25/1887/2007/.

Zhang, Y. C., Liu, Z. X., Shen, C., Fazakerley, A., Dunlop, M., Rème, H., Lucek, E., Walsh, A. P., and Yao, L.: The magnetic structure of an earthward-moving flux rope observed by Cluster in the near-tail, Ann. Geophys., 25, 1471-1476, 2007, http://www.ann-geophys.net/25/1471/2007/.

Zong, Q.-G., Fritz, T. A., Pu, Z. Y., Fu, S. Y., Baker, D. N., Zhang, H., Lui, A. T., Vogiatzis, I., Glassmeier, K.-H., Korth, A., Daly, P. W., Balogh, A., and Reme, H.: Cluster observations of earthward flowing plasmoid in the tail, Geophys. Res. Lett., 31, 18803, doi:10.1029/2004GL020692, 2004. 